\title{
Treatment monitoring of colorectal cancer by integrated analysis of plasma concentration and sequencing of circulating tumor DNA
}

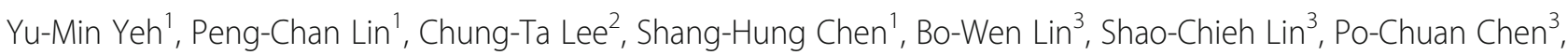
Ren-Hao Chan ${ }^{3}$ and Meng-Ru Shen ${ }^{4,5^{*}}$

\begin{abstract}
Circulating cell-free DNA (cfDNA) analysis is an important tool for cancer monitoring. The patient-specific mutations identified in colorectal cancer (CRC) tissues are usually used to design the cfDNA analysis. Despite high specificity in predicting relapse, the sensitivity in most studies is around $40-50 \%$. To improve this weakness, we designed a cfDNA panel according to the CRC genomic landscape and recurrent-specific mutations. The pathological variants in cfDNA samples from 60 CRC patients were studied by a next-generation sequencing (NGS) method incorporating the dual molecular barcode. Interestingly, patients in the disease positive group had a significantly higher cfDNA concentration than those in the disease negative group. Based on receiver operating characteristic analysis, the cfDNA concentration of $7 \mathrm{ng} / \mathrm{mL}$ was selected into the analytical workflow. The sensitivity in determining the disease status was $72.4 \%$, which represented a considerable improvement on prior studies, and the specificity remained high at $80.6 \%$. Compared to standard imaging and laboratory studies, earlier detection of residual disease and clinical benefits were shown on two cases by this ffDNA assay. We conclude this integrative framework of cfDNA analytical pipeline with a satisfactory sensitivity and specificity could be used in postoperative CRC surveillance.
\end{abstract}

Keywords: Colorectal cancer, Circulating cell-free DNA, Cell-free DNA concentration, Molecular barcode, Nextgeneration sequencing

\section{Main text}

The standard care for CRC patients without distant metastasis is surgical resection of the primary tumor with en bloc removal of the regional lymph nodes. Despite the aggressive use of adjuvant FOLFOX chemotherapy, recurrence develops in a third of patients with stage III

\footnotetext{
* Correspondence: mrshen@mail.ncku.edu.tw

${ }^{4}$ Department of Pharmacology, National Cheng Kung University Hospital, College of Medicine, National Cheng Kung University, Tainan, Taiwan ${ }^{5}$ Department of Obstetrics and Gynecology, National Cheng Kung University Hospital, College of Medicine, National Cheng Kung University, No. 138 Sheng-Li Road, Tainan 704, Taiwan

Full list of author information is available at the end of the article
}

or high-risk stage II disease. Moreover, FOLFOX chemotherapy causes a long-lasting neurotoxicity, negatively impacting the quality of life of survivors $[1,2]$. The development of biomarkers to identify CRC patients who truly have residual disease will allow patients to be treated with appropriate adjuvant chemotherapy and avoid unnecessary treatment-related toxicity in the adjuvant setting.

Circulating cfDNA are short fragmented DNAs which are detectable in plasma [3]. Several studies selected the patient-specific mutations identified in the tumor tissues for cfDNA analysis of blood sample and proved the use

C The Author(s). 2020 Open Access This article is licensed under a Creative Commons Attribution 4.0 International License, which permits use, sharing, adaptation, distribution and reproduction in any medium or format, as long as you give appropriate credit to the original author(s) and the source, provide a link to the Creative Commons licence, and indicate if changes were made. The images or other third party material in this article are included in the article's Creative Commons licence, unless indicated otherwise in a credit line to the material. If material is not included in the article's Creative Commons licence and your intended use is not permitted by statutory regulation or exceeds the permitted use, you will need to obtain permission directly from the copyright holder. To view a copy of this licence, visit http://creativecommons.org/licenses/by/4.0/ The Creative Commons Public Domain Dedication waiver (http://creativecommons.org/publicdomain/zero/1.0/) applies to the data made available in this article, unless otherwise stated in a credit line to the data. 
for postoperative surveillance with different sequencing technologies [4-6]. Overall, the results from these studies [4-6] showed that detection of mutations in cfDNA was strongly associated with inferior recurrence-free survival. The specificity in predicting the relapse was high (96-100\%); however, the sensitivity in predicting relapse was around $40-50 \%$.

To improve the unsatisfactory sensitivity of cfDNA test in predicting tumor recurrence, the important and recurrence-specific CRC mutations identified in our previous study [7] were used to design a cfDNA panel, and a sequencing method incorporating molecular tags was used to detect the low-frequency cfDNA mutations. Moreover, the cfDNA concentration was integrated into the analytical pipeline. This cfDNA analytical workflow showed the satisfactory sensitivity and specificity of determining the disease status were 72.4 and $80.6 \%$, respectively.

\section{Results}

\section{Clinical cohort}

Sixty-nine genes were included in the cfDNA panel (Supplementary Table S1), which was tested in 60 histologically confirmed CRC patients, including 31 patients with no clinical evidence of disease (disease negative group), and 29 patients with clinical evidence of disease (disease positive group). Patients with and without clinical evidence of disease were determined by the results of the most recent imaging studies. The clinicopathological and molecular characteristics were similar between the two groups, except for the stage at initial diagnosis (Supplementary Table S2). cfDNA analysis was tested three times at intervals of 3-6 months for each patient. Because of the loss to follow-up and disease progression leading to death, the results of the 2nd and 3rd cfDNA analysis were available in 54 and 44 patients, respectively (Supplementary Fig. S1).

\section{cfDNA concentrations}

A total of 158 plasma samples were collected. The cfDNA concentrations of these samples are shown in Fig. 1a. In the disease negative group, the majority (84 of 90) of the cfDNA concentrations were less than $10 \mathrm{ng} /$ $\mathrm{mL}$, and the median cfDNA concentration was $4.09 \mathrm{ng} /$ $\mathrm{mL}$. In contrast, the cfDNA concentration in the disease positive group varied from 1.180 to $321 \mathrm{ng} / \mathrm{mL}$, and the median level was $9.47 \mathrm{ng} / \mathrm{mL}$. Patients in the disease
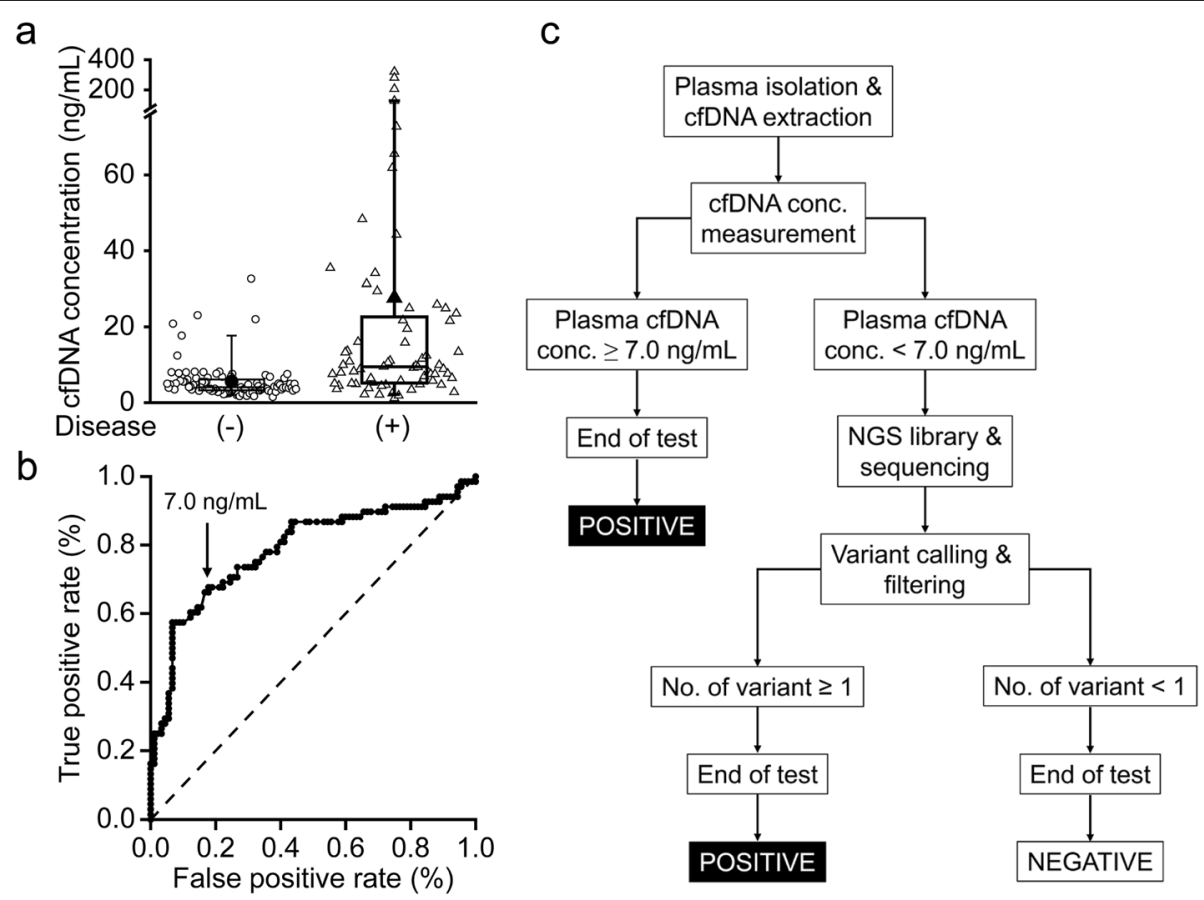

Fig. 1 Proposed workflow of cfDNA analysis for treatment monitoring of CRC patients. a Boxplot displaying the cfDNA levels in plasma collected from patients in the disease negative (open circle; $n=90$ ) and disease positive (open triangle; $n=68$ ) groups at three different time points. The horizontal box boundaries and midline represent the sample quartiles, while the solid circle and triangle indicate the mean of the cfDNA concentrations. The upper and lower whisker denotes the 95th and 5th percentiles, respectively. $\mathbf{b}$ The receiver operating characteristics (ROC) curve of cfDNA concentration in determining the presence or absence of disease. The points in the ROC curve indicate different cfDNA concentrations with corresponding true and false positive rates, from which $7.0 \mathrm{ng} / \mathrm{mL}$ was chosen as the cut-off value for cfDNA concentration. The dotted line is the line of no-discrimination. c The cfDNA concentrations and sequencing results were integrated together in the analytical workflow. For patients with cfDNA concentration $\geq 7.0 \mathrm{ng} / \mathrm{mL}$, the cfDNA test was defined as positive, and for patients with cfDNA concentration $<7.0 \mathrm{ng} / \mathrm{mL}$, the detection of at least one cfDNA variant was required to define a positive cfDNA test 
positive group had a significantly higher cfDNA concentration than those in the disease negative group. ROC analysis was performed to determine an optimal threshold, demonstrating that $7.0 \mathrm{ng} / \mathrm{mL}$ had the best ability to differentiate two groups (Fig. 1b).

\section{Pathological cfDNA mutations}

The library preparation method utilizing molecular barcodes was adopted by the cfDNA assay in order to detect low-frequency variants and improve the sensitivity. The analytical pipeline was shown in Supplementary Fig. S2 and the details of variant calling, annotation and filtering were provided in Supplementary Materials and Methods. When variants with an allele frequency $(\mathrm{AF}) \geq$ $0.1 \%$ were used to determine the variant detected in cfDNA and plasma samples were defined as positive, the cfDNA assay showed a very good sensitivity (0.828); however, the specificity was only 0.484 , and the accuracy was 0.65 (Supplementary Table S3). When the AF cut- off was shifted from 0.1 to $0.2,0.3$, or 0.5 , the sensitivity mildly decreased from 0.828 to $0.793,0.724$, or 0.724 respectively. Concurrently, the improvement of the specificity was observed (from 0.484 to 0.742, 0.806, and 0.806). The best accuracy was 0.767 with the Youden index of 0.53 , which was detected at cut-off levels of 0.3 and $0.5 \%$; thus, $0.5 \%$ was chosen for subsequent analyses.

We proposed a workflow by integrating the concentration and pathological variants of cfDNA (Fig. 1c). When the cfDNA concentration was $\geq 7.0 \mathrm{ng} / \mathrm{mL}$, the test was defined as positive. In patients with a cfDNA concentration $<7.0 \mathrm{ng} / \mathrm{mL}$, one or more variants detected in plasma samples was required to define as positive. Figure 2 showed the result of the 1 st cfDNA analysis using the cfDNA panel in $60 \mathrm{CRC}$ patients. In the disease negative group $(N=31), 4$ patients (Cases $1-4)$ had a cfDNA concentration $>7.0 \mathrm{ng} / \mathrm{mL}$, with no mutation detected in cfDNA samples. Two patients (Cases 8 and 17)

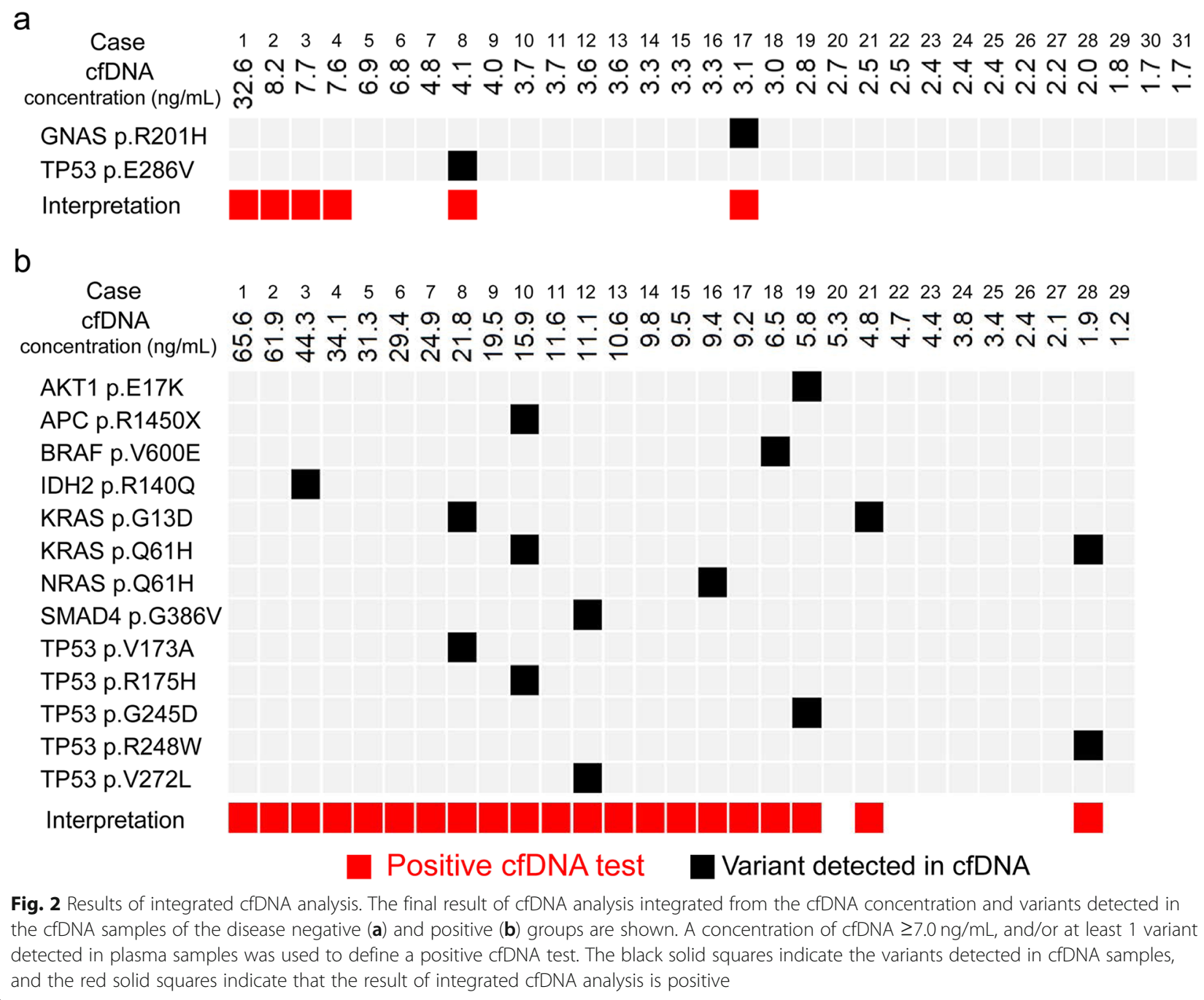

Fig. 2 Results of integrated cfDNA analysis. The final result of cfDNA analysis integrated from the cfDNA concentration and variants detected in the cfDNA samples of the disease negative (a) and positive (b) groups are shown. A concentration of cfDNA $\geq 7.0 \mathrm{ng} / \mathrm{mL}$, and/or at least 1 variant detected in plasma samples was used to define a positive cfDNA test. The black solid squares indicate the variants detected in cfDNA samples, and the red solid squares indicate that the result of integrated cfDNA analysis is positive 


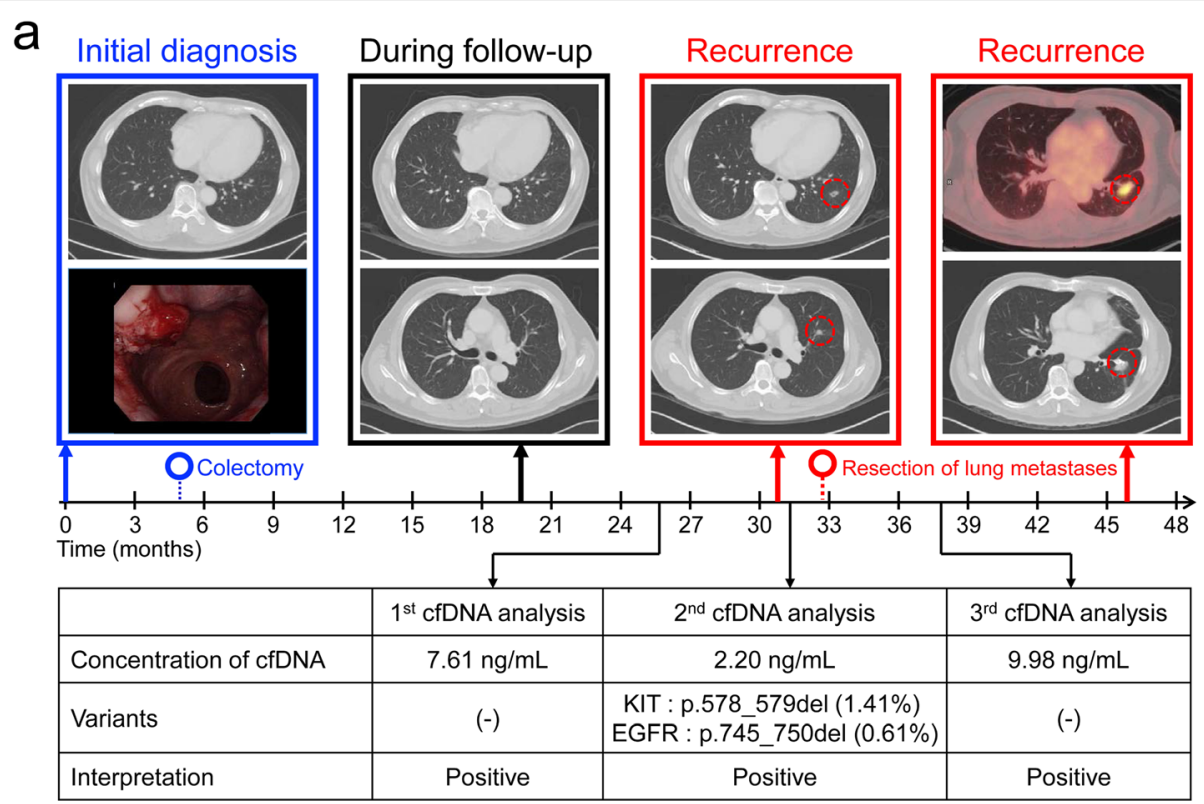

b

Initial diagnosis Recurrence
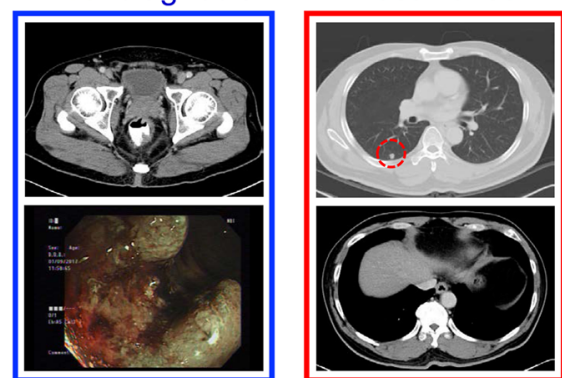

Recurrence

Recurrence
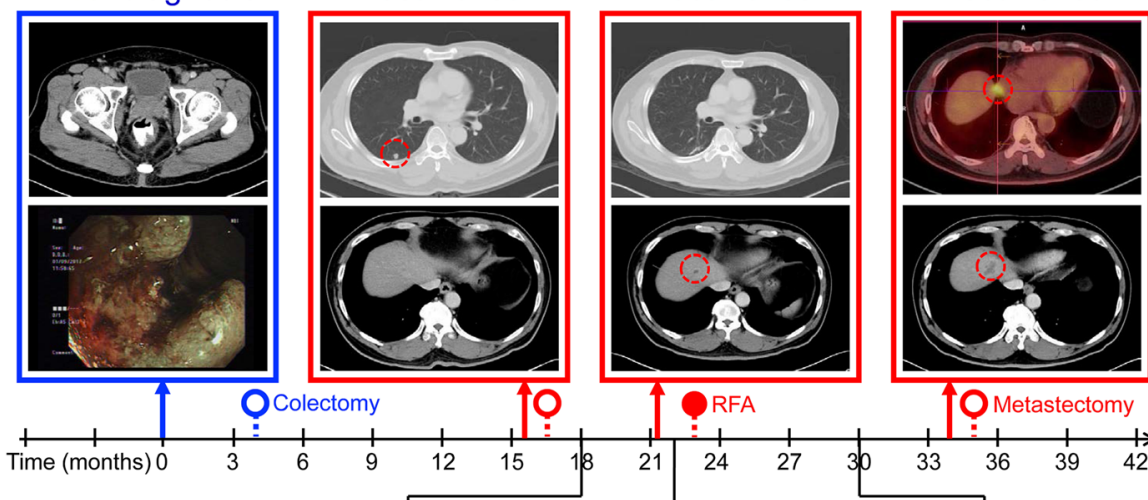

CEA $(\mathrm{ng} / \mathrm{mL}) \quad 9.4 \quad 3.6 \quad 2.3$

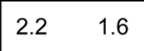

1.32

\begin{tabular}{|l|c|c|c|}
\hline & $1^{\text {st }}$ cfDNA analysis & $2^{\text {nd }}$ cfDNA analysis & $3^{\text {rd }}$ cfDNA analysis \\
\hline Concentration of cfDNA & $5.29 \mathrm{ng} / \mathrm{mL}$ & $10.88 \mathrm{ng} / \mathrm{mL}$ & $7.58 \mathrm{ng} / \mathrm{mL}$ \\
\hline Variants & $(-)$ & $(-)$ & $(-)$ \\
\hline Interpretation & Negative & Positive & Positive
\end{tabular}

Fig. 3 Clinical benefits of the cfDNA assay in two CRC patients. a This patient had a rectal adenocarcinoma, which was treated with pre-operative concurrent chemoradiotherapy followed by a rectum colectomy and post-operative adjuvant chemotherapy. Recurrence with pulmonary metastases was detected 2 years after surgery of the primary tumor. The patient underwent resection of the pulmonary metastases with curative intent, and was treated with systemic chemotherapy for 3 months. The result of the 3rd cfDNA analysis was positive, although the imaging study at that time did not detect any recurrence. Later imaging revealed recurrent tumors around the surgical sutures of the left upper and left lower lobe. b This patient had pathological stage III rectal adenocarcinoma, which was treated with pre-operative concurrent chemoradiotherapy, rectum colectomy, and post-operative adjuvant FOLFOX chemotherapy for 6 months. Recurrence with pulmonary metastasis was detected 1 year after surgery of the primary tumor. The patient underwent complete resection of the pulmonary metastasis, and received salvage chemotherapy with bevacizumab in combination with FOLFIRI. Five months after the metastectomy, the imaging study revealed a new liver metastasis, which was treated with percutaneous radiofrequency ablation (RFA). Following RFA, the cfDNA concentration remained high, which indicated the presence of disease; however, the imaging study at that time did not detect any recurrence. A later CT scan revealed a recurrent tumor in the liver, adjacent to the previous ablation site 
had variants detected in cfDNA samples, but the cfDNA concentration of these patients was $<7.0 \mathrm{ng} / \mathrm{mL}$ (Fig. 2a). According to the proposed workflow, these patients in the disease negative group had positive cfDNA analysis. Tumor recurrence was detected in one (Case 4) of the 6 patients 4 months after the cfDNA analysis.

In the disease positive group, 21 of 29 patients showed positive cfDNA results; 17 patients (Cases 1-17) had a cfDNA concentration $\geq 7.0 \mathrm{ng} / \mathrm{mL}$ (Fig. $2 \mathrm{~b}$ ). Among the 12 patients with a cfDNA concentration $<7.0 \mathrm{ng} / \mathrm{mL}$, variants were detected in 4 patients (Cases 18, 19, 21, and 28). In the disease positive group, the variants were detected most frequently in TP53 $(n=5)$ and KRAS $(n=$ 4) (Supplementary Table S4).

\section{Performance in 2nd and 3rd cfDNA analysis}

The cfDNA analysis was repeated at intervals of 3-6 months. When the results of the 2nd and 3rd cfDNA analysis were pooled, the sensitivity, specificity, and accuracy were $0.821,0.729$, and 0.765 , similar to those observed in the 1st cfDNA analysis (Supplementary Table S5). TP53 and KRAS were still the most common genes with variants detected in the cfDNA (Supplementary Table S6 and S7).

\section{Clinical benefits}

The routine post-treatment surveillance of CRC patients include history, physical examination, carcinoembryonic antigen (CEA) test, and computed tomography (CT) scan. Based on the proposed workflow, two cases showed the clinical benefit of cfDNA assay. Figure 3a outlines the case of a 70-year-old male patient with stage IIIA rectal cancer. After the surgery, lung metastases were detected by CT scan 30 months after initial diagnosis. The patient received resection of the lung lesions with curative intent. The cfDNA test of the 3rd analysis at 38 months was positive based on the high cfDNA concentration $(9.98 \mathrm{ng} / \mathrm{mL})$, but there was no evidence of recurrence by CT scan. However, 8 months later, CT scan showed recurrent tumors around the surgical sutures of the left lung, confirmed by positron emission tomography (PET) scan. Figure 3b highlights another case in the disease positive group. The patient was diagnosed with clinical stage III rectal cancer and the CEA level was $9.4 \mathrm{ng} / \mathrm{mL}$ at initial diagnosis. After the surgery, the CEA level decreased to $2.3 \mathrm{ng} / \mathrm{mL}$ and remained normal in all subsequent tests. During the follow-up, lung and liver metastases were detected. The patient received the surgical resection of lung metastases and percutaneous radiofrequency ablation for liver metastasis. The CT scans at 27 and 30 months did not detect any recurrence but the cfDNA concentration of the 3rd analysis at 30 months was still high $(7.58 \mathrm{ng} / \mathrm{mL})$, indicating a positive test for the disease status. A recurrent tumor in the liver, adjacent to the previous ablation site, was demonstrated by the CT scan 4 months after the 3rd cfDNA analysis. Moreover, the BRAF V600E mutation detected in Case 18 showing a response to the treatment combining a BRAF, MEK and EGFR inhibitor and the disease progression accompanied by the increase of cfDNA concentration observed in many cases in the disease positive group also suggest the potential utility of integrated cfDNA analysis in prediction and monitoring treatment response in advanced colorectal cancer.

\section{Discussion}

This is the first study to integrate the cfDNA concentration into the workflow of cfDNA analysis for monitoring the disease status of CRC patients. The concept of applying cfDNA concentration for postoperative surveillance of CRC patients is supported by a recent study [8]. The tumor heterogeneity and clonal evolution might be the reasons explaining the low sensitivity in prior studies using the patient-specific cfDNA analysis [9]. Tracking multiple mutations across a wide range of genes without personalization is a solution to overcome this limitation [10] and the results of this study are in agreement with this strategy.

\section{Conclusions}

This cfDNA analytical pipeline, integrating the cfDNA concentration and pathological variants detected by an ultra-sensitive sequencing technique, shows a satisfactory sensitivity in postoperative CRC surveillance.

\section{Supplementary Information}

Supplementary information accompanies this paper at https://doi.org/10. 1186/s12943-020-01273-8.

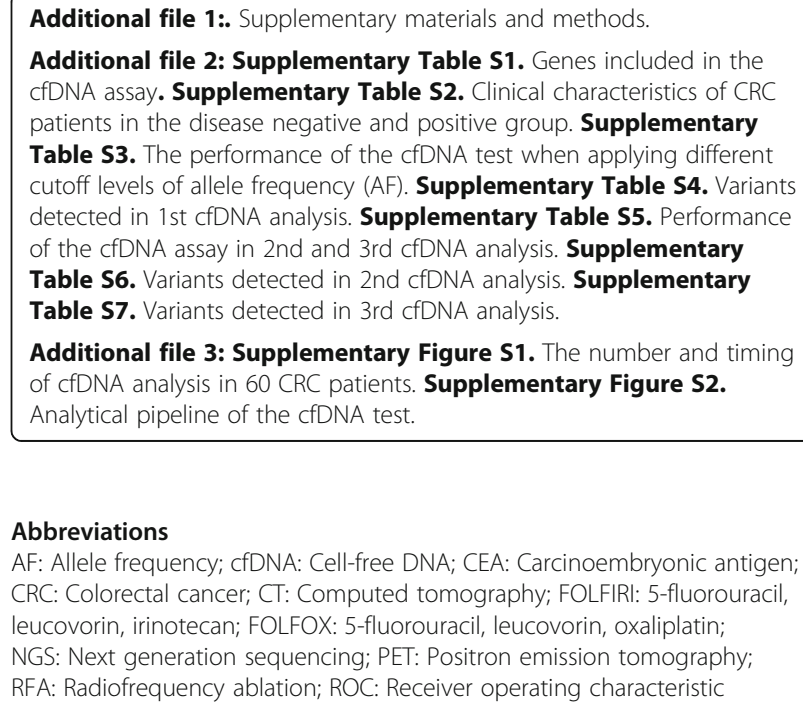

Additional file 3: Supplementary Figure S1. The number and timing of cfDNA analysis in 60 CRC patients. Supplementary Figure S2. Analytical pipeline of the cfDNA test.

\section{Abbreviations}

AF: Allele frequency; cfDNA: Cell-free DNA; CEA: Carcinoembryonic antigen; CRC: Colorectal cancer; CT: Computed tomography; FOLFIRI: 5-fluorouracil, leucovorin, irinotecan; FOLFOX: 5-fluorouracil, leucovorin, oxaliplatin; NGS: Next generation sequencing; PET: Positron emission tomography; RFA: Radiofrequency ablation; ROC: Receiver operating characteristic 


\section{Acknowledgements}

We thank Enago (www.enago.tw) for the English editing. We also thank Kimforest LTD Taiwan for performing NGS experiments and bioinformatics support.

\section{Authors' contributions}

Conception and design: YMY, PCL, MRS; Development of methodology: YMY, PCL, MRS; Acquisition of data: YMY, PCL, CTL, SHC, BWL, SCL, PCC, RHC; Analysis and interpretation of data: YMY, PCL, CTL, MRS; Writing of the manuscript: YMY, MRS; Study supervision: MRS. The author(s) read and approved the final manuscript.

\section{Funding}

This study was supported by the Ministry of Science and Technology (MOST 109-2634-F-006-015 and MOST 109-2634-F-006-023), the Ministry of Health and Welfare (MOHW 109-TDU-B-211-134018 and MOHW 109-TDU-B-211114003), and the Higher Education Sprout Project, Ministry of Education to the Headquarters of University Advancement at National Cheng Kung University.

\section{Availability of data and materials}

All data generated during this study are included in this published article and its supplementary information files.

\section{Ethics approval and consent to participate}

This study was approved by the Institutional Review Board of NCKUH (A-ER108-033), and written informed consent was provided by the participants before enrollment.

\section{Consent for publication}

Written informed consent was obtained for all involved participants.

\section{Competing interests}

The authors declare that they have no competing interests.

\section{Author details}

'Department of Internal Medicine, National Cheng Kung University Hospital, College of Medicine, National Cheng Kung University, Tainan, Taiwan.

${ }^{2}$ Department of Pathology, National Cheng Kung University Hospital, College of Medicine, National Cheng Kung University, Tainan, Taiwan. ${ }^{3}$ Department of Surgery, National Cheng Kung University Hospital, College of Medicine, National Cheng Kung University, Tainan, Taiwan. ${ }^{4}$ Department of Pharmacology, National Cheng Kung University Hospital, College of Medicine, National Cheng Kung University, Tainan, Taiwan. ${ }^{5}$ Department of Obstetrics and Gynecology, National Cheng Kung University Hospital, College of Medicine, National Cheng Kung University, No. 138 Sheng-Li Road, Tainan 704, Taiwan.

Received: 5 August 2020 Accepted: 21 October 2020

Published online: 26 October 2020

\section{References}

1. Pachman DR, Qin R, Seisler DK, Smith EM, Beutler AS, Ta LE, et al. Clinical course of Oxaliplatin-induced neuropathy: results from the randomized phase III trial N08CB (alliance). J Clin Oncol. 2015;33(30):3416-22.

2. Kidwell KM, Yothers G, Ganz PA, Land SR, Ko CY, Cecchini RS, et al. Longterm neurotoxicity effects of oxaliplatin added to fluorouracil and leucovorin as adjuvant therapy for colon cancer: results from National Surgical Adjuvant Breast and bowel project trials C-07 and LTS-01. Cancer. 2012;118(22):5614-22.

3. Fan HC, Blumenfeld YJ, Chitkara U, Hudgins L, Quake SR. Analysis of the size distributions of fetal and maternal cell-free DNA by paired-end sequencing. Clin Chem. 2010:56(8):1279-86.

4. Diehl F, Schmidt K, Choti MA, Romans K, Goodman S, Li M, et al. Circulating mutant DNA to assess tumor dynamics. Nat Med. 2008;14(9):985-90

5. Tie J, Wang Y, Tomasetti C, Li L, Springer S, Kinde I, et al. Circulating tumor DNA analysis detects minimal residual disease and predicts recurrence in patients with stage II colon cancer. Sci Transl Med. 2016;8(346):346ra92.

6. Reinert T, Henriksen TV, Christensen E, Sharma S, Salari R, Sethi H, et al. Analysis of Plasma Cell-Free DNA by Ultradeep Sequencing in Patients With Stages I to III Colorectal Cancer. JAMA Oncol. 2019;5(8):1124-31.
7. Lin PC, Yeh YM, Wu PY, Hsu KF, Chang JY, Shen MR. Germline susceptibility variants impact clinical outcome and therapeutic strategies for stage III colorectal cancer. Sci Rep. 2019;9(1):3931.

8. Fleming CA, O'Leary DP, Wang J, Redmond HP. Association of Observed Perioperative Cell-Free DNA Dynamics With Early Recurrence in Patients With Colon Cancer. JAMA Surg. 2020;155(2):168-70.

9. McGranahan N, Swanton C. Biological and therapeutic impact of intratumor heterogeneity in cancer evolution. Cancer Cell. 2015;27(1):15-26.

10. Bronkhorst AJ, Ungerer V, Holdenrieder $\mathrm{S}$. The emerging role of cell-free DNA as a molecular marker for cancer management. Biomol Detect Quantif. 2019;17:100087.

\section{Publisher's Note}

Springer Nature remains neutral with regard to jurisdictional claims in published maps and institutional affiliations.
Ready to submit your research? Choose BMC and benefit from:

- fast, convenient online submission

- thorough peer review by experienced researchers in your field

- rapid publication on acceptance

- support for research data, including large and complex data types

- gold Open Access which fosters wider collaboration and increased citations

- maximum visibility for your research: over $100 \mathrm{M}$ website views per year

At $\mathrm{BMC}$, research is always in progress.

Learn more biomedcentral.com/submissions 